\title{
Limberg flap reconstruction for sacrococcygeal pilonidal sinus disease with and without acute abscess: Our experience and a review of the literature
}

\author{
Catherine J. Sinnott, Laurence T. Glickman \\ Long Island Plastic Surgical Group, Garden City, NY, USA
}

Background The efficacy of Limberg flap reconstruction for pilonidal sinus with acute abscess remains unclear. This study aimed to compare outcomes after Limberg flap reconstruction for pilonidal sinus disease with and without acute abscess. A secondary objective was to perform a review of the literature on the topic.

Methods A retrospective chart review was conducted of all patients who underwent excision and Limberg flap reconstruction for pilonidal sinus from 2009 to 2018. Patient demographics, wound characteristics, and complication rates were reviewed and analyzed.

Results Group 1 comprised 19 patients who underwent Limberg flap reconstruction for pilonidal sinus disease without acute abscess and group 2 comprised four patients who underwent reconstruction for pilonidal sinus disease with acute abscess. The average defect size after excision was larger in group 2 than group $1\left(107.7 \pm 60.3 \mathrm{~cm}^{2}\right.$ vs. $61.4 \pm 33.8 \mathrm{~cm}^{2}$, respectively). There were no recurrences, seromas or cases of flap necrosis postoperatively. There was only one revision surgery needed for evacuation of a postoperative hematoma in group 1. There were comparable rates of partial wound dehiscence treated by local wound care, hematoma, need for revision surgery and minor infection between group 1 and group 2.

Conclusions Limberg flap reconstruction for pilonidal sinus in the setting of acute abscess is a viable option with outcomes comparable to that for disease without acute abscess. This practice will avoid the pain and cost associated with a prolonged local wound care regimen involved in drainage of the abscess prior to flap reconstruction.

Keywords Pilonidal sinus / Surgical flap / Abscess
Correspondence: Catherine J. Sinnott Long Island Plastic Surgical Group, 999 Franklin Avenue, Garden City, NY 11530, USA

Tel: +1-617 285-5505

Fax: +1-516-629-3887

E-mail: catherine.sinnott@gmail.com

\section{INTRODUCTION}

Sacrococcygeal pilonidal sinus disease is a common and debilitating condition, with 26 cases per 100,000 patients occurring annually [1]. Young, hirsute males are typically affected by this condition. Pilonidal disease was originally thought to be a congenital condition caused by abnormal skin in the gluteal cleft [2]; however, it is now believed to be an acquired condition caused by the presence of hair in the gluteal cleft [3]. This hair causes an inflammatory reaction, producing chronic draining si- 


\section{Fig. 1. Patient with pilonidal sinus disease without abscess}
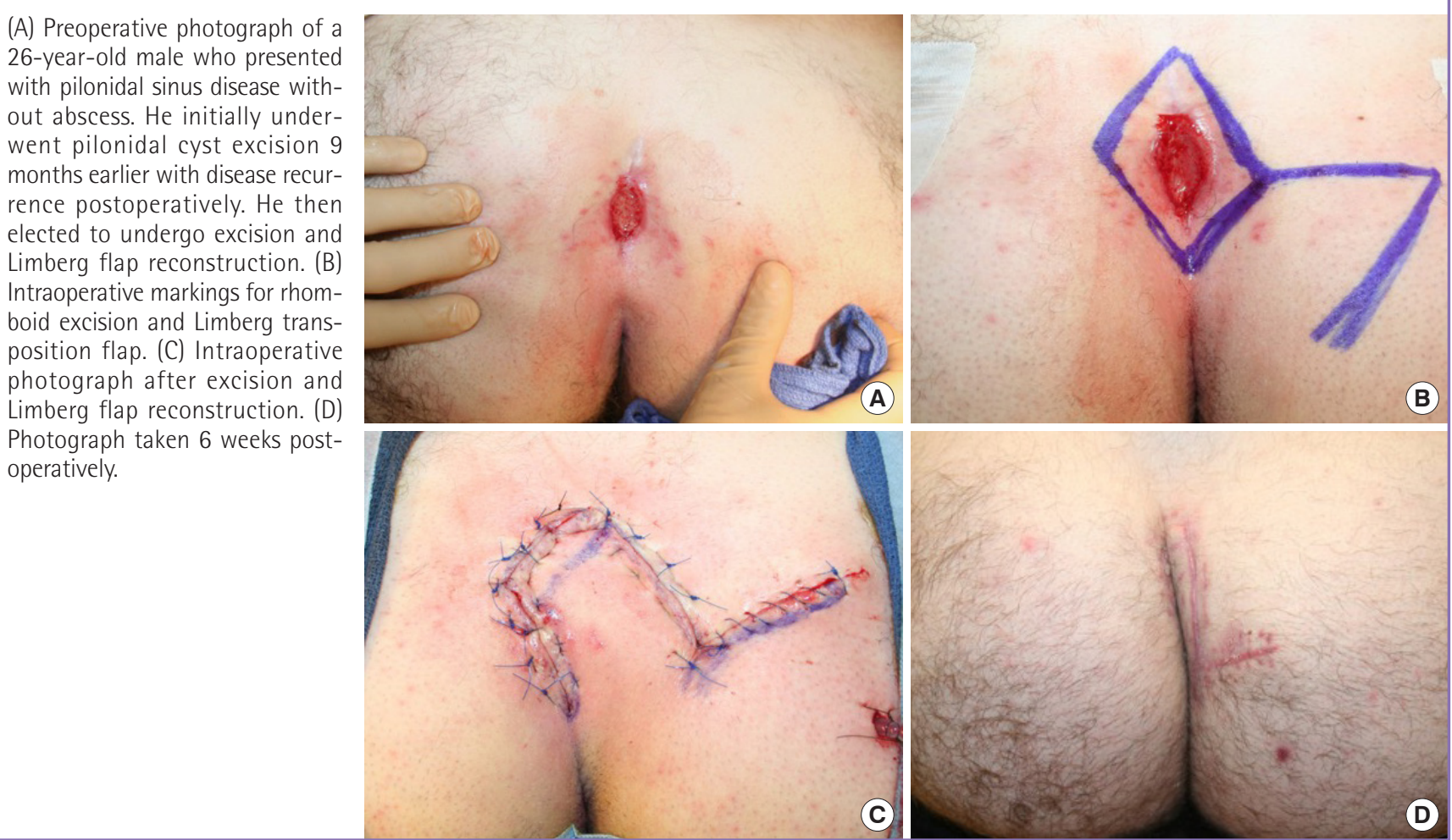

nuses and tracts (Fig. 1A) and often secondary infection and abscess formation (Fig. 2A). Surgical methods of treating pilonidal disease include incision and drainage, laying open of the sinus tract, excision and healing by secondary intention, excision and primary closure and excision with advancement or transposition flap closure. Treatment of pilonidal disease upon initial clinical presentation is usually by conservative methods of incision and drainage or excision with healing by secondary intention. While some patients never have a recurrence after conservative management of the first episode, many go on to have recurrent disease with chronic draining sinuses and abscesses, producing significant pain, distress and discomfort. For these patients, the recommended treatment is wide excision of all involved tissue, including the underlying sinus tract and any lateral extensions. When the surrounding healthy tissue is closed primarily, the tissues are placed under significant tension and wound breakdown and disease recurrence is common. Therefore, closure with a transposition flap, such as the Limberg flap, is usually recommended to reduce tension on the wound.

Treatment for pilonidal sinus disease associated with acute abscess usually involves incision and drainage or abscess excision versus wide local excision and healing by secondary intention. Flap reconstruction is usually then performed several weeks later after resolution of infection. The recurrence rate after initial surgical management varies widely and depends on clinical pre- sentation and whether it is acute or chronic and the surgical treatment modality.

The Limberg flap, initially described by Alexander Limberg in 1948, is a transposition flap for coverage of a rhomboid-shaped defect with opposite sides of equal length and opposite medial and lateral angles of $120^{\circ}$ and superior and inferior angles of $60^{\circ}$ $[4,5]$. The innovative design of the Limberg flap allows for tension-free closure of the rhomboid-shaped defect and the donor site. Several studies have suggested that rhomboid excision and closure with Limberg transposition flap is the treatment of choice for chronic sacrococcygeal pilonidal sinus disease [6-11]. These studies suggest that the Limberg flap produces superior results compared to other surgical treatment options, with regard to disease recurrence, flap failure and complications. However, few studies have evaluated the effectiveness of the Limberg flap reconstruction for pilonidal sinus disease with acute abscess. It is usually advised to drain abscesses and wait for resolution of active infection prior to performing flap reconstruction [12]. The purpose of this study was to describe our results using Limberg transposition flap reconstruction for sacrococcygeal pilonidal sinus disease with and without acute abscess. A secondary objective of this study was to perform a review of the literature on the topic of flap reconstruction and other surgical treatment modalities for sacrococcygeal pilonidal sinus disease in the setting of acute abscess. 
Fig. 2. Patient with pilonidal sinus disease with abscess

\begin{abstract}
(A) Preoperative photograph of a 23-year-old male who presented with pilonidal sinus disease with abscess. He underwent excision of pilonidal sinus disease in the past and had recurrence with abscess formation. (B) Intraoperative photograph after extended rhomboid excision of pilonidal sinus disease with abscess. (C) Intraoperative photograph after Limberg flap reconstruction of rhomboid defect. (D) Photograph taken 3 years postoperatively.
\end{abstract}
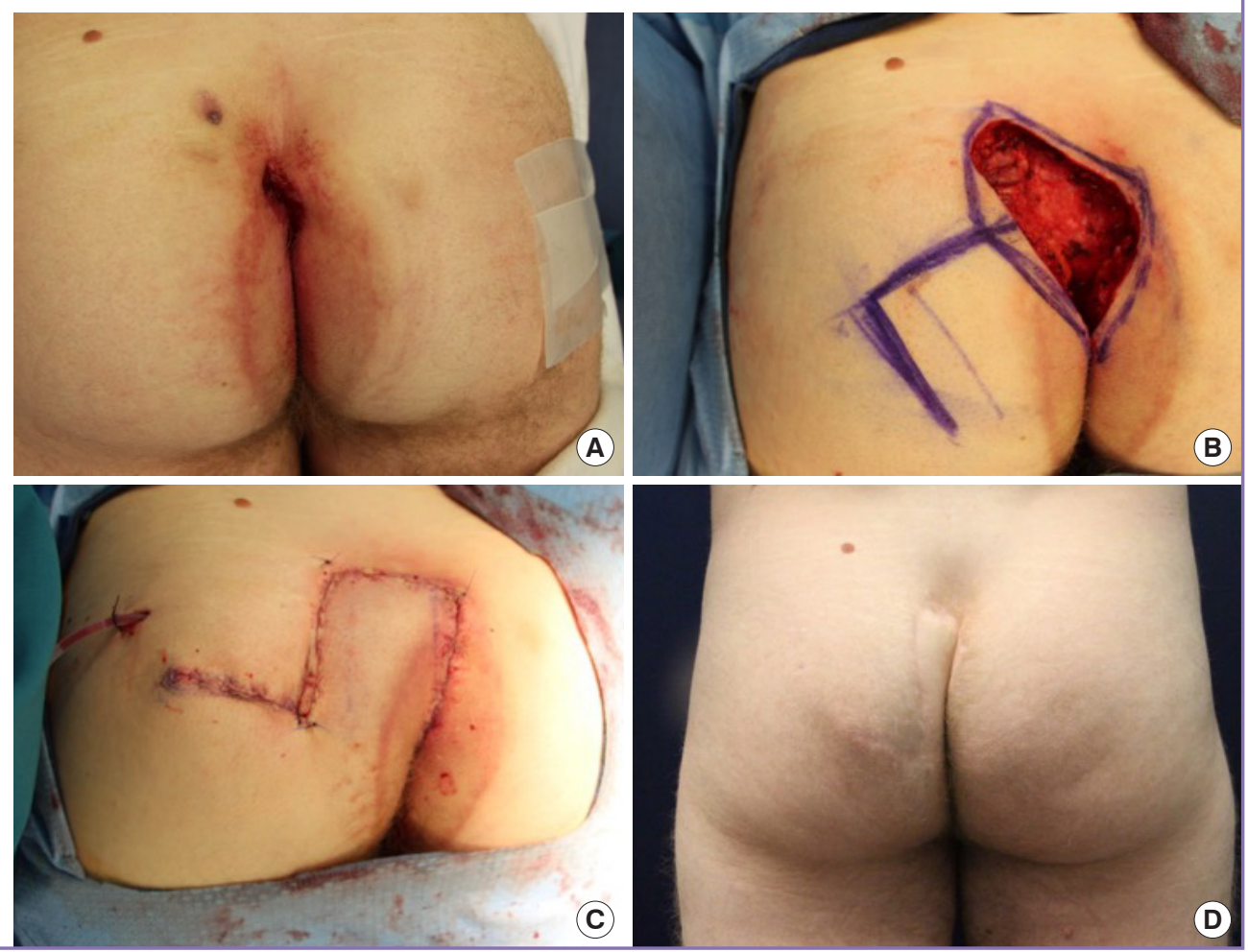

\section{METHODS}

Approval for this study was obtained through NYU Winthrop Institutional Review Board (IRB No. 17425). Informed consent was obtained from all patients to participate in this study and for the educational and academic use of any photographic images taken in the pre-, peri- or postoperative period. A retrospective chart review was conducted of all operations for pilonidal sinus disease performed by the senior author (LTG) from 2009 to 2018. Patients who underwent Limberg transposition flap reconstruction for sacrococcygeal pilonidal sinus disease were included. Patients who underwent other surgical procedures for treatment of sacrococcygeal pilonidal sinus disease were excluded. Patients were assigned to one of two groups based on clinical presentation, with group 1 made up of patients having Limberg flap reconstruction for pilonidal sinus disease without acute $a b$ scess and group 2 made up of patients having Limberg flap reconstruction for sacrococcygeal pilonidal sinus disease with acute abscess. Pilonidal sinus disease with abscess was defined as disease in the acute setting characterized by the presence of a painful fluctuant mass or by the presence of cellulitis draining purulent fluid, indicating a recently decompressed fluctuant abscess. Demographic and clinical data were reviewed and recorded, including, age, sex, body mass index (BMI), past medical history of diabetes, smoking history and duration of symptoms prior to excision. Operative characteristics were also recorded and analyzed, including, wound defect size after excision, suture material and number of layers used to close the wound, as well as the duration of drain use. Outcomes were assessed by analyzing the rates of postoperative wound dehiscence, infection, seroma, hematoma, necrosis, recurrence and the need for revision surgery. Continuous variables were reported as the mean \pm standard deviation (SD) and categorical variables and complication rates were reported as percentages.

Surgery was performed on all patients in prone position under general anesthesia. A rhomboid-shaped area of skin was marked over the sacrococcygeal pilonidal sinus and included any lateral extensions (Fig. 1B). The angles of the excised area of skin were $120^{\circ}$ at the medial and lateral aspects and $60^{\circ}$ at the superior and inferior aspects. The skin and subcutaneous tissue of the rhomboid-shaped area was incised and elevated at the level of the presacral fascia and above the gluteus maximus fascia. All affected tissue was excised (Fig. 2B). A Limberg flap was then designed with limb lengths comparable in size to the rhomboid-shaped defect. Hemostasis was achieved and the wound was copiously irrigated. A 7-mm Jackson-Pratt drain was brought out through a small stab incision. The Limberg flap was then transposed into the defect. The flap and donor site were closed primarily in three layers using polydioxanone (PDS), Monocryl, and Prolene suture (Figs. 1C, 2C) and a sterile dressing was applied. Patients 
were discharged and drains and sutures were removed postoperatively. Patients were seen in clinic postoperatively on a weekly or bi-weekly basis, or until it was confirmed that complete wound healing without complications had occurred (Figs. 1D, 2D).

The vascular territory of the Limberg flap is supplied by the lateral sacral and superior gluteal arteries, which arise from the posterior division of the internal iliac artery. The lateral sacral arteries enter the anterior sacral foramina in the pelvis and exit the pelvis through the corresponding posterior sacral foramina, supplying the skin and muscles of the posterior surface of the sacrum and forming abundant vascular communication with branches of the superior gluteal artery over the gluteus maximus muscle [13]. It is therefore possible to design the Limberg flap as a random-pattern cutaneous flap which can be transposed medially to fill the defect remaining after pilonidal sinus excision. Designed as a random-pattern cutaneous flap and not a perforator flap, the blood supply to the Limberg flap does not come from a single recognized artery, but from many small unnamed vessels, the majority of which are derived from lateral sacral and superior gluteal arteries.

\section{RESULTS}

Twenty-three patients underwent Limberg flap reconstruction for sacrococcygeal pilonidal disease during the study period with a mean age of $25.3 \pm 12.0$ years (mean \pm SD) and a mean BMI of $27.8 \pm 4.0 \mathrm{~kg} / \mathrm{m}^{2}$ (Table 1$)$. Twenty patients $(87.0 \%)$ were male, $17.4 \%$ (4 patients) were active smokers and $8.7 \%$ (2 patients) were diabetic. The average defect size after excision of pilonidal sinus disease in all patients was $69.5 \pm 41.8 \mathrm{~cm}^{2}$ and the average duration of drain use before removal was $5.2 \pm 3.1$ days. Over a mean follow-up time of 16.3 months, there was one revision surgery required for evacuation of hematoma. Partial wound dehiscence occurred in seven patients and was treated

Table 1. Demographic and clinical characteristics of patients having Limberg flap reconstruction

\begin{tabular}{|lccc|}
\hline Characteristic & Total & Group 1 & Group 2 \\
\hline No. of patients & 23 & 19 & 4 \\
Age (yr) & $25.3 \pm 12.0$ & $24.6 \pm 12.2$ & $29.0 \pm 12.2$ \\
Male sex & $87.0(20)$ & $84.2(16)$ & $100(4)$ \\
BMl $\left(\mathrm{kg} / \mathrm{m}^{2}\right)$ & $27.8 \pm 4.0$ & $27.6 \pm 4.2$ & $28.8 \pm 3.1$ \\
Smoking & $17.4(4)$ & $15.8(3)$ & $25.0(1)$ \\
Diabetes & $8.7(2)$ & $10.5(2)$ & 0 \\
Defect size $\left(\mathrm{cm}^{2}\right)$ & $69.5 \pm 41.8$ & $61.4 \pm 33.8$ & $107.8 \pm 60.3$ \\
Drain time (day) & $5.2 \pm 3.1$ & $5.6 \pm 3.2$ & $3.2 \pm 1.9$ \\
Follow up (mon) & $16.3 \pm 24.1$ & $17.1 \pm 26.2$ & $12.7 \pm 11.5$ \\
\hline Values are presented as mean $\pm \mathrm{SD}$ or percent (number). & \\
BMl, body mass index. & & & \\
\hline
\end{tabular}

with local wound care only and minor infection occurred in one patient and was treated with oral antibiotics only. Prior to Limberg flap reconstruction, all patients had recurrent disease and had undergone one or more treatments of incision and drainage or excision with healing by secondary intention or excision and primary closure for sacrococcygeal pilonidal sinus disease.

Group 1 was made up of 19 patients who underwent Limberg transposition flap reconstruction for pilonidal sinus disease without abscess and group 2 was comprised of four patients who underwent Limberg transposition flap reconstruction for pilonidal sinus disease with abscess (Table 1). Demographic and clinical characteristics were similar between the two groups. The average defect size was larger in group 2 compared to group $1\left(107.8 \pm 60.3 \mathrm{~cm}^{2}\right.$ vs. $61.4 \pm 33.8 \mathrm{~cm}^{2}$, respectively). This finding supports our belief that a wider area of resection is required to remove all sinuses and tracks in pilonidal sinus disease with concomitant abscess formation prior to Limberg transposition flap reconstruction. The duration of drain use was longer in group 1 compared to group 2 (5.6 \pm 3.2 days vs. $3.2 \pm 1.9$ days).

There were no cases of recurrent disease, seroma or flap necrosis in either group 1 or group 2 (Table 2). Partial wound dehiscence requiring treatment with local wound care only was comparable between group 1 and group $2(31.6 \%[n=6]$ vs. $25.0 \%$ $[\mathrm{n}=1])$, as was the rate of hematoma and the need for revision surgery $(5.3 \%[n=1]$ vs. $0 \%$, respectively). The rate of minor infection requiring oral antibiotics only was higher in group 2 compared to group 1 ( $25.0 \%$ vs. $0 \%)$. There were no cases of major infection requiring intravenous antibiotics or operative intervention in either group 1 or group 2.

\section{DISCUSSION}

Many studies have shown that Limberg flap reconstruction is one of the most effective methods of surgical treatment for sacrococcygeal pilonidal sinus disease [6-11]. However, few studies have examined the use of Limberg flap reconstruction for pi-

Table 2. Complications and outcomes after Limberg flap reconstruction

\begin{tabular}{|lccc|}
\hline Complication & Total & Group 1 & Group 2 \\
\hline No. of patients & 23 & 19 & 4 \\
Hematoma & $4.3(1)$ & $5.3(1)$ & 0 \\
Seroma & 0 & 0 & 0 \\
Dehiscence & $30.4(7)$ & $31.6(6)$ & $25.0(1)$ \\
Necrosis & 0 & 0 & 0 \\
Revision surgery & $4.3(1)$ & $5.3(1)$ & 0 \\
Infection & $4.5(1)$ & 0 & $25.0(1)$ \\
Recurrence & 0 & 0 & 0 \\
\hline Values are presented as percent (number). & & \\
\hline
\end{tabular}


lonidal sinus disease with acute abscess. It is usually advised to drain abscesses and wait for resolution of active infection prior to performing flap reconstruction for pilonidal sinus disease with acute abscess [12]. However, the results of this study indicate that Limberg transposition flap reconstruction may be a viable surgical treatment modality for pilonidal sinus disease with acute abscess formation. Outcomes and postoperative complication rates were similar in group 1 and group 2 . There were no cases of disease recurrence, major dehiscence, major infection, flap necrosis or seroma requiring revision surgery. The only complications to have occurred were one hematoma in group 1 requiring operative evacuation, partial wound dehiscence, treated with local wound care only and occurring at comparable rates in group 1 and group 2, and one case of minor infection occurring in group 2, treated with oral antibiotics only. Appropriate management of sacrococcygeal pilonidal sinus disease requires making an accurate diagnosis and determining if abscess formation is present. If abscess formation is present then traditional management would involve either incision and drainage or wide local excision with healing by secondary intention, followed by flap reconstruction at a later date when infection had cleared. The findings in this study, however, suggest that although pilonidal sinus disease with acute abscess requires a more extensive resection than disease without abscess, Limberg flap reconstruction can be performed concomitantly with wide local excision with acceptable outcomes compared to flap reconstruction for pilonidal sinus disease without acute abscess. Limberg flap reconstruction for sacrococcygeal pilonidal sinus disease with acute abscess may reduce the pain and discomfort, cost and protracted treatment course associated with incision and drainage and waiting several weeks for resolution of infection prior to Limberg flap reconstruction.

A secondary objective of this study was to perform a review of the literature on the use of flap reconstruction and other surgical treatment modalities for sacrococcygeal pilonidal sinus disease in the setting of acute abscess. Fahrni et al. [14] showed that limited abscess excision versus wide local excision of pilonidal sinus disease with acute abscess followed by healing by secondary intention was associated with higher rate of recurrence ( $38.9 \%$ vs. $13.3 \%$, respectively; $\mathrm{P}=0.0001$ ). Furthermore, in cases of chronic pilonidal sinus disease without acute abscess limited excision followed by healing by secondary intention was associated with a higher, although not significantly different, recurrence rate (27.6\%), compared to wide local excision followed by either healing by secondary intention $(11.3 \%, \mathrm{P}=$ $0.068)$ or primary closure $(26.8 \%, \mathrm{P}=1.000)$. Ciftci et al. [15] showed that oblique excision and primary closure versus incision and drainage for acute pilonidal abscess was associated with lower recurrence of pilonidal sinus disease $(6.0 \%$ vs. $78.8 \%$, respectively; $\mathrm{P}<0.001)$. Kanat et al. [16] showed that unroofing and curettage of pilonidal abscess versus Karydakis flap reconstruction following drainage and antibiotics produced comparable complication rates ( $7.1 \%$ vs. $8.0 \%$, respectively) and recurrence rates (3.5\% vs. $4.0 \%)$. However, Karydakis flap reconstruction was preferred because of a shorter treatment duration and higher patient comfort. In contrast to our study, Karydakis flap reconstruction in this study was performed for pilonidal sinus disease after incision and drainage of abscess and resolution of infection. Also, in contrast to our study, Muzi et al. [17] showed that Limberg flap reconstruction or tension free primary closure for pilonidal disease with acute abscess was successful (without complication, recurrence or failure) in only $60.0 \%$ of cases versus $86.8 \%$ of cases without acute abscess $(\mathrm{P}<0.0001)$, making the presence of acute abscess formation a negative prognostic factor.

Certain limitations of this study have to be considered when evaluating its impact on clinical practice. The non-randomized, retrospective design, and its higher probability of selection bias, as well as the lower number of patients and statistical power to detect significant differences are important limitations in this study. Although this study is limited by the small number of patients belonging to group 2, precluding statistical analysis and statistical comparison between the two groups, the importance of this study lies in that it identifies trends and similarities in outcomes between Limberg flap reconstruction for pilonidal sinus disease with and without abscess. Our study was underpowered to detect significance between the two groups. Further prospective, randomized studies are needed to determine if Limberg flap reconstruction is equally effective for pilonidal sinus disease with acute abscess as it is for pilonidal sinus disease without abscess.

Limberg flap reconstruction for sacrococcygeal pilonidal sinus in the setting of acute abscess may be a viable method of surgical treatment with outcomes comparable to that for pilonidal sinus without abscess. Although it is usually advised to drain acute abscesses associated with pilonidal sinus disease and wait for resolution of active infection prior to flap reconstruction, the results of this study show that Limberg flap reconstruction may be equally effective for pilonidal sinus disease with acute abscess formation. Partial wound dehiscence was the most common complication to occur after Limberg flap reconstruction for sacrococcygeal pilonidal sinus disease with or without acute abscess; however, all cases were managed with local wound care and did not require operative intervention. Limberg flap reconstruction for sacrococcygeal pilonidal sinus disease with acute abscess will hasten resolution of disease and return to normal 
living and reduce the pain, discomfort and cost associated with the prolonged wound care regimen involved in incision and drainage and waiting several weeks for resolution of infection prior to flap reconstruction.

\section{NOTES}

\section{Conflict of interest}

No potential conflict of interest relevant to this article was reported.

\section{Ethical approval}

The study was approved by the NYU Winthrop Institutional Review Board (IRB No. 17425) and performed in accordance with the principles of the Declaration of Helsinki. Written informed consents were obtained.

\section{Patient consent}

The patients provided written informed consent for the publication and the use of their images.

\section{Author contribution}

Study concept and design: all authors. Data acquisition: Sinnott CJ. Data analysis and interpretation: Sinnott CJ. Drafting of the manuscript: Sinnott CJ. Critical revision of the manuscript for important intellectual content: all authors. Statistical analysis: Sinnott CJ. Administrative, technical, or material support: all authors. Study supervision: all authors. Approval of final manuscript: all authors.

\section{ORCID}

Catherine J. Sinnott https://orcid.org/0000-0002-3489-6031

Laurence T. Glickman https://orcid.org/0000-0001-91057229

\section{REFERENCES}

1. Ferri FF. Ferri's clinical advisor 2018. Philadelphia: Elsevier; 2018.

2. Hodges RM. Pilo-nidal sinus. Boston Med Surg J 1880;103: 485-86.

3. Karydakis GE. Easy and successful treatment of pilonidal sinus after explanation of its causative process. Aust N Z J Surg 1992;62:385-9.

4. Limberg AA. Mathematical principles of local plastic procedures on the surface of the human body. Leningrad: Medg- iz; 1948.

5. Limberg AA. Design of local flaps. In: Gibson T, editor. Modern trends in plastic surgery. Vol. 2. London: Butterworth; 1966. p. 38-61.

6. Akca T, Colak T, Ustunsoy B, et al. Randomized clinical trial comparing primary closure with the Limberg flap in the treatment of primary sacrococcygeal pilonidal disease. $\mathrm{Br} \mathrm{J}$ Surg 2005;92:1081-4.

7. Abu Galala KH, Salam IM, Abu Samaan KR, et al. Treatment of pilonidal sinus by primary closure with a transposed rhomboid flap compared with deep suturing: a prospective randomised clinical trial. Eur J Surg 1999;165:468-72.

8. Ertan T, Koc M, Gocmen E, et al. Does technique alter quality of life after pilonidal sinus surgery? Am J Surg 2005; 190 : 388-92.

9. Dass TA, Zaz M, Rather A, et al. Elliptical excision with midline primary closure versus rhomboid excision with Limberg flap reconstruction in sacrococcygeal pilonidal disease: a prospective, randomized study. Indian J Surg 2012; 74:305-8.

10. Tavassoli A, Noorshafiee S, Nazarzadeh R. Comparison of excision with primary repair versus Limberg flap. Int J Surg 2011;9:343-6.

11. Arslan K, Said Kokcam S, Koksal H, et al. Which flap method should be preferred for the treatment of pilonidal sinus? A prospective randomized study. Tech Coloproctol 2014; 18:29-37.

12. Steele SR, Perry WB, Mills S, et al. Practice parameters for the management of pilonidal disease. Dis Colon Rectum 2013;56:1021-7.

13. Gray H, Lewis WH. Anatomy of the human body. 20th ed. Philadelphia: Lea \& Febiger; 1918.

14. Fahrni GT, Vuille-Dit-Bille RN, Leu S, et al. Five-year followup and recurrence rates following surgery for acute and chronic pilonidal disease: a survey of 421 cases. Wounds 2016;28:20-6.

15. Ciftci F, Abdurrahman I, Tosun M, et al. A new approach: oblique excision and primary closure in the management of acute pilonidal disease. Int J Clin Exp Med 2014;7:5706-10.

16. Kanat BH, Bozan MB, Yazar FM, et al. Comparison of early surgery (unroofing-curettage) and elective surgery (Karydakis flap technique) in pilonidal sinus abscess cases. Ulus Travma Acil Cerrahi Derg 2014;20:366-70.

17. Muzi MG, Milito G, Cadeddu F, et al. Randomized comparison of Limberg flap versus modified primary closure for the treatment of pilonidal disease. Am J Surg 2010;200:9-14. 